\title{
On Sasakian manifolds with vanishing contact Bochner curvature tensor
}

\author{
By Izumi Hasegawa and Toshiyuki Nakane
}

(Received November 21, 1979; Revised January 23, 1980)

\section{§ 1. Introduction.}

Recently, S. I. Goldberg and M. Okumura [3] proved

THEOREM A. Let $M$ be an n-dimensional compact conformally flat Riemannian manifold with constant scalar curvature $R$. If the length of the Ricci tensor is less than $R / \sqrt{n-1}, n \geqq 3$, then $M$ is a space of constant curvature.

For a Kaehlerian manifold, Y. Kubo [7] proved

THEOREM B. Let $M$ be a real n-dimensional Kaehlerian manifold with constant scalar curvature $R$ whose Bochner curvature tensor vanishes. If the length of the Ricci tensor is not greater than $R / \sqrt{n-2}, n \geqq 4$, then $M$ is a space of constant holomorphic sectional curvature.

Note that the square of the length of the Ricci tensor is greater than or equal to $R^{2} / n$, so the Ricci tensor has been "pinched".

We have the following remarks [5] on Theorem B.

REMARK 1. The condition with respect to the length of the Ricci tensor can be replaced by

$$
\text { (*) } \quad R_{a b} R^{a b} \leqq \frac{R^{2}}{n-2} .
$$

REMARK 2. Moreover the condition $(*)$ can be replaced by the best condition

$$
R_{a b} R^{a b}<\frac{n^{3}-2 n^{2}+32}{(n+2)^{2}(n-4)^{2}} R^{2} \quad \text { for } \quad n>4 .
$$

REMARK 3. In paticular, when $M$ is of dimension 4, if the scalar curvature does not vanish, then $M$ is of constant holomorphic sectional curvature.

The purpose of this paper is to obtain the theorems, analogous to the above theorems, for a Sasakian manifold with vanishing contact Bochner curvature tensor.

TheOREm 1. Let $M$ be a $(2 n+1)$-dimensional Sasakian manifold with 
constant scalar curvature $R$ whose contact Bochner curvature tensor vanishes. If the square of the length of the $\eta$-Einstein tensor is less than

$$
\frac{(n-1)(n+2)^{2}(R+2 n)^{2}}{2 n(n+1)^{2}(n-2)^{2}}, n \geqq 3,
$$

then $M$ is a space of constant $\phi$-holomorphic sectional curvature.

Theorem 2. Let $M$ be a 5-dimensional Sasakian manifold with constant scalar curvature whose contact Bochner curvature tensor vanishes. If the scalar curvature is not -4 , then $M$ is a space of constant $\phi$-holomorphic sectional curvature.

\section{$\S 2 . \quad$ Preliminaries.}

Let $M$ be a $(2 n+1)$-dimensional Sasakian manifold with the Riemannian metric $g_{i j}$ and an almost contact structure $(\phi, \xi, \eta)$ satisfying

$$
\begin{aligned}
& \phi_{a}{ }^{i} \phi_{j}{ }^{a}=-\delta^{i}{ }_{j}+\eta_{j} \xi^{i}, \phi_{a}{ }^{i} \xi^{a}=0, \eta_{a} \phi_{i}{ }^{a}=0, \eta_{a} \xi^{a}=1, \\
& N_{i j}{ }^{k}+\left(\partial_{i} \eta_{j}-\partial_{j} \eta_{i}\right) \xi^{k}=0, \phi_{i j}=\frac{1}{2}\left(\partial_{i} \eta_{j}-\partial_{j} \eta_{i}\right), \\
& g_{a b} \phi_{i}{ }^{a} \phi_{j}{ }^{b}=g_{i j}-\eta_{i} \eta_{j}, \xi^{i}=g^{i a} \eta_{a},
\end{aligned}
$$

where $N_{i j}{ }^{k}$ is the Nijenhuis tensor with respect to $\phi_{i j}$ and $\partial_{i}=\partial / \partial x^{i}$ is the partial differential operator with respect to the local coorsinate $\left(x^{i}\right)$. In view of the last equation we shall write $\eta^{i}$ instead of $\xi^{i}$ in the sequel.

In the following, let $R_{h i j k}, R_{i j}, R$ and $\nabla_{i}$ denote the Riemannian curvature tensor, the Ricci tensor, the scalar curvature and the operator of covariant differentiation with respect to $g_{i j}$ respectively. It is well known that in a $(2 n+1)$-dimensional Sasakian manifold we have the following identities :

$$
\begin{aligned}
& \nabla_{i} \eta^{j}=\phi_{i}{ }^{j}, \\
& \nabla_{i} \phi_{j}{ }^{a}=-g_{i j} \eta^{k}+\delta_{i}{ }^{k} \eta_{j}, \\
& \eta^{a} R_{a i j k}=\eta_{k} g_{i j}-\eta_{j} g_{i k}, \\
& \eta^{a} R_{a i}=2 n \eta_{i}, \\
& R_{h i j}{ }^{a} \phi_{a k}-R_{h i k}{ }^{a} \phi_{a j}=\phi_{i j} g_{h k}-\phi_{i k} g_{h j}+g_{i j} \phi_{h k}-g_{i k} \phi_{h j}, \\
& \phi^{a b} R_{a i j b}=R_{i}{ }^{a} \phi_{a j}-(2 n-1) \phi_{i j}, \\
& R_{i}{ }^{a} \phi_{a j}+R_{j}{ }^{a} \phi_{a i}=0, \\
& \phi^{a b} R_{a b i j}=-2 R_{i}{ }^{a} \phi_{a j}+2(2 n-1) \phi_{i j} .
\end{aligned}
$$


DEFinition . 1. If a tensor $T_{i_{1} \cdots i_{p}}{ }^{j_{1} \cdots j_{q}}$ of a Sasakian manifold $M$ satisfies

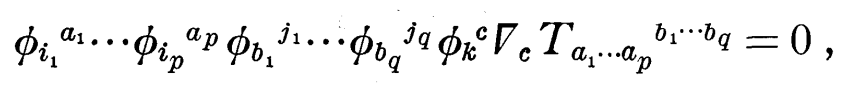

then we say that the tensor $T_{i_{1} \cdots i_{p}}{ }^{j_{1} \cdots j_{q}}$ of $M$ is $\eta$-parallel. If the Riemannian curvature tensor of a Sasakian manifold $M$ is $\eta$-parallel, then we say that $M$ is locally $\phi$-symmetric.

Definition 2. If the Ricci tensor $R_{i j}$ of a Sasakian manifold $M$ satisfies

$$
R_{i j}=\left(\frac{R}{2 n}-1\right) g_{i j}-\left(\frac{R}{2 n}-2 n-1\right) \eta_{i} \eta_{j},
$$

then we say that $M$ is an $\eta$-Einstein mainfold.

We define the $\eta$-Einstein tensor $S_{i j}$ by

$$
S_{i j}=R_{i j}-\left(\frac{R}{2 n}-1\right) g_{i j}+\left(\frac{R}{2 n}-2 n-1\right) \eta_{i} \eta_{j} .
$$

If the $\eta$-Einstein tensor $S_{i j}$ of a Sasakian manifold $M$ vanishes, then $M$ is an $\eta$-Einstein mainfold.

(2.1), (2.3), (2.5) and the second Bianchi identity

$$
\nabla_{l} R_{h i j k}+\nabla_{h} R_{i l j k}+\nabla_{i} R_{l h j k}=0
$$

give

$$
\eta^{a} \nabla_{a} R_{h i j k}=0 .
$$

From this fact and brief computations, we have

Lemma 1 [4]. A Sasakian manifold $M$ is locally $\phi$-symmetric if and only if the Riemannian curvature tensor of $M$ satisfies

$$
\begin{gathered}
\nabla_{l} R_{h i j k}=-\phi_{l}{ }^{a}\left(\eta_{h} R_{a i j k}+\eta_{i} R_{h a j k}+\eta_{j} R_{h i a k}+\eta_{k} R_{h i j a}\right) \\
\quad+\eta_{h}\left(\phi_{l k} g_{i j}-\phi_{l j} g_{i k}\right)+\eta_{i}\left(\phi_{l j} g_{h k}-\phi_{l k} g_{h j}\right) \\
\quad+\eta_{j}\left(\phi_{l i} g_{h k}-\phi_{l h} g_{i k}\right)+\eta_{k}\left(\phi_{l h} g_{i j}-\phi_{l i} g_{h j}\right) .
\end{gathered}
$$

Lemma 2. Let $M$ be a $(2 n+1)$-dimensional Sasakian manifold. The Ricci tensor of $M$ is $\eta$-parallel if and only if the Ricci tensor satisfies the following identity:

$$
\nabla_{k} R_{i j}=-\phi_{k}{ }^{a}\left(R_{a j} \eta_{i}+R_{a i} \eta_{j}\right)+2 n\left(\phi_{k j} \eta_{i}+\phi_{k i} \eta_{j}\right) .
$$

From brief computations, we see that the Ricci tensor is $\eta$-parallel if a Sasakian manifold $M$ is locally $\phi$-symmetric.

For a $(2 n+1)$-dimensional Sasakian manifold, we define the contact Bochner curvature tensor $B_{h i j k}$ of $M$ by 


$$
\begin{aligned}
B_{h i j k} & =R_{h i j k}-\frac{1}{2(n+2)}\left(R_{i j} g_{h k}-R_{i k} g_{h j}+g_{i j} R_{h k}-g_{i k} R_{h j}\right. \\
& -R_{i j} \eta_{h} \eta_{k}+R_{i k} \eta_{h} \eta_{j}-\eta_{i} \eta_{j} R_{h k}+\eta_{i} \eta_{k} R_{h j}+H_{i j} \phi_{h k} \\
& \left.-H_{i k} \phi_{h \jmath}+\phi_{i j} H_{h k}-\phi_{i k} H_{h j}-2 H_{h i} \phi_{j k}-2 \phi_{h i} H_{j k}\right) \\
& +\frac{R-6 n-8}{4(n+1)(n+2)}\left(g_{i j} g_{h k}-g_{i k} g_{h j}\right) \\
& +\frac{R+4 n^{2}+6 n}{4(n+1)(n+2)}\left(\phi_{i j} \phi_{h k}-\phi_{i k} \phi_{h j}-2 \phi_{h i} \phi_{j k}\right) \\
& -\frac{R+2 n}{4(n+1)(n+2)}\left(g_{i j} \eta_{h} \eta_{k}-g_{i k} \eta_{h} \eta_{j}+\eta_{i} \eta_{j} g_{h k}-\eta_{i} \eta_{k} g_{h j}\right),
\end{aligned}
$$

where $H_{i j}=R_{i}^{a} \phi_{a j}$.

Taking the covariant differentiation of $(2.16)$ and contraction, we have the following

Lemma 3. Let $M$ be a Sasakian manifold with vanishing contact Bochner curvature tensor. If the scalar curvature is constant, then the Ricci tensor of $M$ is $\eta$-parallel.

Also, we know the following

Lemma 4 . Let $M$ be a Sasakian manifold with vanishing contact Bochner curvature tensor. If $M$ is an $\eta$-Einstein manifold, then $M$ is a space of constant $\phi$-holomorphic sectional curvature.

M. Okumura [8] proved

Lemma 5. Let $c_{i}, i=1,2, \cdots, m$, be real numbers satisfying

$$
\sum_{i=1}^{m} c_{i}=0 \text { and } \sum_{i=1}^{m} c_{i}{ }^{2}=k^{2} \quad(k=\text { constant } \geqq 0) .
$$

Then we have

$$
-\frac{m-2}{\sqrt{m(m-1)}} k^{3} \leqq \sum_{i=1}^{m} c_{i}{ }^{3} \leqq \frac{m-2}{\sqrt{m(m-1)}} k^{3} .
$$

\section{$\S 3$. Proofs of theorems.}

Proof of Theorem 1.

Let $M$ be a $(2 n+1)$-dimensional Sasakian manifold. Assume that the contact Bochner curvature tensor vanishes, then we have

$$
\begin{aligned}
R_{a b c d} R^{a d} R^{b c}=\frac{2}{n+2} R_{a}^{b} R_{b}{ }^{c} R_{c}{ }^{a}+\frac{(2 n+1) R-2\left(5 n^{2}+8 n+2\right)}{2(n+1)(n+2)} R_{a b} R^{a b} \\
+\frac{-R^{3}+2(5 n+4) R^{2}+12 n^{2} R-8 n^{3}(2 n+1)}{4(n+1)(n+2)}
\end{aligned}
$$


Since the contact Bochner curvature tensor vanishes and the scalar curvature is constant, from Lemma 3, the Ricci tensor is $\eta$-parallel. Therefore we have (2.15) by virtue of Lemma 2. Then, from this fact and the Ricci identity, we obtain

$$
\begin{aligned}
R_{a b c d} R^{a d} R^{b c}-R_{a}^{b} R_{b}^{c} R_{c}^{a} & =R^{b c} g^{a d}\left(\nabla_{b} \nabla_{a} R_{c d}-\nabla_{a} \nabla_{b} R_{c d}\right) \\
& =-R_{a b} R^{a b}+4 n R-4 n^{2}(2 n+1) .
\end{aligned}
$$

Substituting (3.1) into (3.2), we have

$$
\begin{aligned}
& 4 n(n+1) R_{a}^{b} R_{b}{ }^{c} R_{c}^{a}+[4 n(4 n+5)-2(2 n+1) R] R_{a b} R^{a b}+R^{3} \\
& \quad-2(5 n+4) \mathrm{R}^{2}+4 n\left(4 n^{2}+9 n+8\right) R-8 n^{2}(2 n+1)\left(2 n^{2}+5 n+4\right)=0 .
\end{aligned}
$$

From the definition of the $\eta$-Einstein tensor $S_{i j}$, we have $S_{i}{ }^{a} \phi_{a}{ }^{j}=\phi_{i}{ }^{a} S_{a}{ }^{j}$. Moreover we see that

$$
\begin{aligned}
& \text { trace } S=S_{a}^{a}=0, \\
& \text { trace } S^{2}=S_{a b} S^{a b}=R_{a b} R^{a b}-\frac{1}{2 n} R^{2}+2 R-4 n^{2}-2 n \geqq 0, \\
& \text { trace } S^{3}=S_{a}^{b} S_{b}{ }^{c} S_{c}^{a}=R_{a}^{b} R_{b}{ }^{c} R_{c}{ }^{a}-3\left(\frac{R}{2 n}-1\right) R_{a b} R^{a b}+\frac{1}{2 n^{2}} R^{3} \\
& -\frac{3}{n} R^{2}+6(n+1) R-4 n(n+1)(2 n+1) \\
& =R_{a}^{b} R_{b}{ }^{c} R_{c}{ }^{a}-3\left(\frac{R}{2 n}-1\right) S_{a b} S^{a b}-\frac{1}{4 n^{2}} R^{3}+\frac{3}{2 n} R^{2}-3 R \\
& -2 n(2 n+1)(2 n-1) .
\end{aligned}
$$

$M$ is an $\eta$-Einstein mainfold if and only if trace $S^{2}$ vainshes.

Substituting (3.5) and (3.6) in (3.3), we have

$$
2 n(n+1) \text { trace } S^{3}+(n+2)(R+2 n) \text { trace } S^{2}=0 \text {. }
$$

We put $f^{2}=$ trace $S^{2}(f \geqq 0)$. From $S_{i}{ }^{a} \eta_{a}=0$ and the comutativity of $S_{i}{ }^{j}$ and $\phi_{i}{ }^{j}$, we see that the characteristic roots of $S_{i}{ }^{j}$ are $c_{1}, \cdots, c_{n}, c_{1}, \cdots, c_{n}$ and 0 . Combining this fact with Lemma 5, we have

$$
-\frac{n-2}{\sqrt{2 n(n-1)}} f^{3} \leqq \text { trace } S^{3} \leqq \frac{n-2}{\sqrt{2 n(n-1)}} f^{3} \text {. }
$$

Applying the above inequality, (3.7) yields the following inequality

$$
\begin{aligned}
& f^{2}\left[(n+2)(R+2 n)-\frac{2 n(n+1)(n-2)}{\sqrt{2 n(n-1)}} f\right] \leqq 0 \\
& \quad \leqq f^{2}\left[(n+2)(R+2 n)+\frac{2 n(n+1)(n-2)}{\sqrt{2 n(n-1)}} f\right]
\end{aligned}
$$


By the assumption of Theorem 1, we see that $f^{2}=0$, that is $M$ is an $\eta$ Einstein mainfold. From Lemma 4, Theorem 1 has been proved.

REMARK. In Theorem 1, the condition with respect to the square of the length of the $\eta$-Einstein tensor is equivalent to

$$
\begin{aligned}
R_{a b} R^{a b} & <\frac{n^{3}-n^{2}+4}{2(n+1)^{2}(n-2)^{2}} R^{2}-\frac{2\left(n^{4}-3 n^{3}-6 n^{2}+4 n+8\right)}{(n+1)^{2}(n-2)^{2}} R \\
& +\frac{2 n^{2}\left(2 n^{4}-3 n^{3}-7 n^{2}+8 n+12\right)}{(n+1)^{2}(n-2)^{2}} .
\end{aligned}
$$

Proof of Theorem 2.

Let $M$ be a 5-dimensional Sasakian manifold with constant scalar curvature whose contact Bochner curvature tensor vanishes. We see that trace $S^{3}=0$ since the characteristic roots of $S_{i}{ }^{j}$ are $c,-c, c,-c$ and 0 . From (3.7) we have $4(R+4)$ trace $S^{2}=0$. Therefore we have Theorem 2 by virtue of Lemma 4 .

\section{References}

[1] S. I. GoldberG: On conformally flat spaces with definite Ricci curvature, Kōdai Math. Sem. Rep., 21 (1969), 226-232.

[2] S. I. GOLDBERG: On conformally flat spaces with definite Ricci curvature II, Kōdai Math. Sem. Rep., 27 (1976), 445-448.

[3] S. I. Goldberg and M. OKumura: Conformally flat manifolds and a pinching problem on the Ricci tensor, Proc. Amer. Math. Soc., 58 (1976), 234-236.

[4] I. HASEgAWA: Sasakian manifolds with $\eta$-parallel contact Bochner curvature tensor, J. Hokkaido Univ. Ed. Sect. II A, 29 (1979), 1-5.

[5] I. HASEgawA and T. NAKANE: Remarks on Kaeherian manifolds with vanishing Bochner curvature tensor, to appear.

[6] I. HASEgAwA and T. NAKANE: On Sasakian manifolds with vanishing contact Bochner curvature tensor II, to appear.

[7] Y. Kubo: Kaehlerian manifolds with vanishing Bochner curvature tensor, Kōdai Math. Sem. Rep., 28 (1976), 85-89.

[8] M. OKumura: Hypersurfaces and a pinching problem on the second fundamental tensor, Amer. J. Math. 96 (1974), 207-212.

Mathematics Laboratory

Sapporo College

Hokkaido University of

Education 